auf die hier behandelte Frage beziehen, und deren frühere Einsicht mir durch die Zeitverhältnisse verwehrt war. Die wichtigsten von ihnen sind in dem Nachtrag angefühirt.

BULiLN-NEWTON beschreibt als Rxetomya eine Charakterform des westafrikanischen Eozäns, die auf dieses beschränkt ist und sich von Ägypten bis Deutsch-Südwest findet. Es scheinen sich also gewisse malakologische Sondermerkmale der westafrikanischen Eozänfauna zu ergeben. - Die Brannersche Arbeit ist die erste, zusammenfassende Darstellung der brasilianischen Geologie. Die schöne Karte gibt eine klare Vorstellung der Verbreitung der marinen Sedimente im Bereich der brasilianischen Masse. Es scheint, als wenn marine Schichten bei der Zusammensetzung des Perm eine größere Rolle spielen wie man bisher annahm, so daß im Perm das Meer im Bereich der brasilianischen Masse vielleicht eine erheblich größere Ausdehnung besessen hat, wie auf S. 68 dieser Arbeit angenommen ist.

\title{
Die Fortschritte der geologischen Forschung in Argentinien und einigen Nachbarstaaten während des Weltkriegs. ${ }^{1}$ )
}

\author{
Von H. Gerth.
}

Obwohl der Weltkrieg auch an den Ländern Südamerikas nicht spurlos rorübergegangen ist, hat doch die geologische Erforschuing dieses Kontinents während der Kriegsjahre ganz gewaltige Fortschritte gemacht. Vor allem ist es die geologische Landesuntersuchung in Argentinien, die in den ersten Kriegsjahren eine äußerst rege Tätigkeit entwickelt hat. Später, als sich dann die Folgen des Krieges auch in diesen fernen Iändern mehr und mehr geltend machten und die Tätigkeit im Felde nicht mehr-möglich war, hat intensive Laboratoriumsarbeit eine überaus stattliche Anzahl von Publikationen hervorgebracht. Sie haben unsere Kenntnis vom geologischen Aufbau dieses Landes und von seinen Mineralschätzen um ein gutes Teil bereichert und nicht wenig Ergebnisse gezeitigt, die auch ein allgemeines Interesse beanspruchen.

Anales del Ministerio de Agricultura: Sección Geología, Mineralogía y Minoría:

1. Pastore, F., Estudio geológico y petrográfico de la Sierra del Morro. Tomo XI, No. 2. 1915 .

2. KaId fu, J., La geología de las Sierras de la Provincia de Buenos Aires y sus relaciones con las montañas de Sud-Africa y los Andes. Tomo XI, No. 3. 1916.

3. Stappenbeok, R., Geología de la falda oriental de la Cordillera del Plata (Provincia de Mendoza). Tomo XIT, No. 1. 1917.

4. Bonarelui, G., Tierra del Fuego y sus Turberas. Tomo XIT, No. 3. 1917.

1) Vgl. das frühere Referat in Bd. VI dieser Zeitschr. 1915. 
5. Wrommaxr, R., Geología y Hidrología de Bahía Blanca y sus alrededores (Provincia de Buenos Aires). Tomo XIII, No. 1. 1918.

6. Sobrat, J. M., Estudio petrográfico de algunas rocas argentinas. Tomo XIII, No. 2. 1918.

7. WIChmans, Ru. Fstudios geológicos y hidrológicos de la región comprendida entre Boca del Rio Negro, San Antonio y Choel Choel, Tomo XIII, No. 3. 1918.

8. - Contribución a la Geología de la región comprendida entre el Rio Negro y Arroyo Valcheta. Tomo XIII, No. 4. 1919.

9. Nágera, J. J., La Sierra Baya. Tomo XIV, No. 1. 1919.

Außerdem kurze Tätigkeitsberichte der Sección Geología in den jährlichen Memorias der Dirección de Minas.

Bolletines de la Dirección General de Minas, Geológía y Hidrología:

10. Snteglitz, O., Contribución a la petrografía de la Precordillera y del Pie de Palo. No. 10. 1914.

11. GRotber, P., Informe sobre las causas que ham producido las crecientes del Rio Colorado (Territorios del Neuquen y la Pampa) en 1914. No. 11. 1916.

12. BeDer, R., Los yacimientos de los minerales de wolfram en la República Argentina. No. 12. 1916.

13. Rassmus, J., Rasgos geológicos generales de las Sierras Pampeanas. No. '13. 1916.

14. BeDER, Ru., Estudios geológicos e hidrogeológicos en los alrededores de Villa Dolores (Provincia de Córdoba). No. 14. 1916.

15. Windhausen, A., Los yacimientos petrolíféros de la Zona Andina (Provincia de Mendoza y Territorio del Neuquen). No. 15.1916.

16. BEDER, R., Contribución a la cristalografía del diópsido de las cales cristalinas de la Sierra de Córdoba. No. 16. 1916.

17. Russmus, J., Investigaciones de la estructura tectónica de la cuenca imbrifera del rio de la Rioja con motivo de la diminución del caudal de dicho río. No. 17. 1918.

18. GRombER, P., Estratigrafía del Dogger en la República Argentina; estudio syntetico comparativo. No. 18. 1918.

19. STaPrenbeck, R., Los Yacimientos de minerales y rocas de aplicación en la República Argentina. No. 19. 1918.

20. Wichmans, R., Investigaciones hidrogeológicas en Puerto Deseado y sus alrededores (Territorio Nacional de Santa Cruz) con motivo de la provisión de.agua al citado pueblo. No. 20. 1919.

21. Harsen, J., Contribución al estudio de la petrografía del T'erritorio Naciona] de Misiones. No. 21. 1919.

22. Nagera, J. J., Nota geológica sobre el Cerro San Agustin. No. 22. 1919. Boletin de la Academia nacional de ciencias de Córdoba (República Argentina):

23. Bodensender, G., El nevado de Famatina. Tomo XXI. 1916.

24. Rumavi, E., Estudio geológico de la Sierra Chica entre Ongamira y Dalares. Tomo XXIII. 1918.

25. Beder, R., y Windhausen, A., Sobre la presencia del devónico en la parte media de la República del Paraguay. Tomo XXIII. 1918.

26. Frengunul, J., Notas preliminares sobre la constitución geológica del súbsuelo en la cuenca de Cordoba. Tomo XXIII. 1918.

27. Domring, A., Nota al estudio sobre la constitución geológica del subsuelo en la cuenca de Cordoba del doctor J. FrengunLLI. Tomo XXIII. 1918.

28. Casteliatios, A., Observaciones preliminares sobre el pleistoceno de la Provincia de Córdoba. Bolet. Acad. Nacion. d. Cienc. Tomo XXIIT. 1918.

29. Windhausen, A., Rasgos de la historia geológica de la planicie costanera en la Patagonia septentrional. Tomo XXIII. 1918. 
30. Wrohmann, R., Sobre la constitución geológica del territorio del Rio Negro y la Región vecina. Sociedad Argentina de Ciencias Naturales Buenos Aires.

31. - El estado actual del Monte Hermoso. Physis, Tomo II. Buenos Aires. 1916.

32. - Las capas con Dinosaurios en la costa sur del Rio Negro, Frente a General Roca. Physis. Tomo II. Buenos Aires. 1916.

33. WALTHER, K., Lineas fundamentales de la estructura geológica de la republica o. del Uruguay. Revis. Instit, de Agronomía de Montivideo. 1915 ${ }^{1}$ ).

34. Winduausen, A., The problem of the Cret.-tert. boundary in South America and the stratigraphic position of the San Jorge-Formation in Patagonia. Americ. Journ. of Science 44. 1918.

35. Casteluatio,

Der südamerikanische Kontinent setzt sich bekanntlich aus zwei ganz verschiedenen Strukturelementen zusammen: dem Kordillerenstreifen im Westen und der sogenannten brasilianischen Masse, die den östlichen Hauptteil des Kontinents ausmacht. Während die Geschichte des Kordillerengebiets verhältnismäßig jung ist (es handelt sich um eine mesozoische Geósynllinale, die erst in jüngster Zeit aufgefaltet wurde), liegt die der brasilianischen Massse weiter zurück, und verhält sich ihrer Erforschung gegenüber viel spröder, als die ihre Entwicklung noch offen zur Schau tragenden Kordilleren. In den jüngeren Perioden der Erdgeschichte spielte die brasilianische Masse nur die passive Rolle eines alten Kontinents, dessen Randpartien immer mehr unter terrestrischen Aufschïttungen begraben werden, und nur das gewaltige Ereignis der Kordillerenfaltung zieht mit seinen Ausläufern auch ihn noch einmal in Mitleidenschaft. Er wird zerstückelt und einzelne Teile dex alten unter den jungen Aufschüttungen begrabenen Masse in Gestalt der pampinen Sierren und Vorkordilleren wieder ans Tageslicht gebracht. Sie sind es, die uns in Argentinien ihren Bau und ihre Geschichte verfolgen lassen.

Sie lassen schon heute deutlich zwei Hauptphasen in der orogenetischen Entwicklung dieser alten Kontinentalmasse erkennen. Die ältere liegt weit zurück in frühpaläozoischer oder vorpaläozoischer Zeit, und durch sie wurde die erste Anlage eines atlantischen Kontinents geschaffen, der vermutlich ausgedehnte Teile des heutigen Südamerika mit Südafrika verband. Im Westen und Süden wurde er begrenzt von einem paläozoischen Geosynklinalmeer, das zuweilen weit nach Osten über die alte Masse übergreift. Die Ablagerungen des Kambrium und Silur bleiben im Westen zurück und sind nur aus den östlichsten Kordillerenzügen bekannt. Dort fand KeIDEL (Memoria 1915, S.42) in Schiefern von bathyaler Fazies eine individuenreiche Trilobitenfauna, die dem oberen Kambrium angehört. Im Unterdevon drang das Meer dann weit über die abradierte Oberfläche der alten Masse vor. In Bra-

1) S. a. WaxtheR, K. Über den gegenwärtigen Stand der geologischen Erforschung der Republik Uruguay. Zeitșehr. d. deutsch. wissenseh. Ver. Buenos Aires. 1919. 
silien ist die paläozoische Sedimentbedeckung nur noch in Fetzen erhalten; ein neues Bindeglied zwischen diesen und den zusammenhängenden paläozoisehen Bildungen der östlichen Kordilleren ist das von Bẹder (23) entdeckte Devonvorkommen inmitten der Republik Paraguay, zwischen den Flüssen Piribebuy und Manduviva, dessen Fossilien von WindhausEN beschrieben worden sind. Es handelt sich auch hier um die für die Südhalbkugel charakteristische Fauna des höheren Unterdevons, die kürzlich von ClarkE ${ }^{1}$ ) aus Brasilien, den Vorkordilleren und von den Falklandsinseln zusammenfassend behandelt wurde und die schon früher namentlich aus Bolivien bekannt geworden war. ClakkE weist erneut auf die außerordentlich engen Beziehungen dieser Fauna zu. der der Bokkeveldserie des Kapgebirges hin. Er kommt zu dem Ergebnis, daß der eigentümliche Charakter des antarktischen Devonmeeres nur bei der Annahme größerer Kontinentalmassen zu erklären ist, die es vom mediterranen Gebiete trennten. In Südostbrasilien, Uruguay und den östlichen pampinen Sierren fehlen die paläozoischen Ablagerungen über den älteren Bildungen. $O b$ wir es in diesem Gebiet mit einer zweiten jüngeren Faltung zu tun haben, die BEDER (13) mit den kaledonischen Bewegungen der Nordhalblsugel vergleichen möchte, müssen weitere Forschungen entscheiden. Die nicht näher dëutbaren Fossilspuren in den kontakt- und dynamometamorph veränderten Marmoren, die in der Sierra de Córdoba in den älteren Gesteinen eingefaltet, scheinen mir keineswegs beweisend für ein frühsilurisches Alter derselben. Allem Anschein nach haben wir es aber in diesen Gegenden mit einer paläozoischen Landmasse zu tun, an deren Südküste uns die Sierren der Provinz Buenos Aires führen. Hier lagern sich wieder mächtige paläozoische Sedimente diakordant auf die alten metamorphen Gesteine. Außer Pflanzenresten $(9,22)$ konnten bis jetzt keine Fossilien in ihnen gefunden werden, doch weist KEIDEL (2) auf die große Ähnlichkeit in Gliederung und petrographischer Beschaffenheit mit den Bildungen des Kapgebirges in Südafrika hin, und zwar vergleicht er die liegenden Quarzite mit dem Tafelbergsandstein und die darüber folgenden Schiefer und Sandsteine mit der Bokkeveldserie. Gegen Ende des Paläozoikums tritt die brasilianische Masse in die. zweite Phase ihrer Entwicklung. Entlang ihrem West- und Südrand schaffen orogenetische Vorgänge ein Saumgebirge, das zunächst in meridionaler Richtung verläuft und dann wie die heutigen Kordilleren nach Osten umbiegt. Das weite, von diesem Gebirgszuge nach Nordosten umschlossene Gebiet wird endgültig als Kontinentalmasse herausgehoben. In der argentinischen Vorkordillere der Provinzen Mendoza und San Juan und in der südlicheren der pampinen Sierren der Provinz Buenos Aires treten Stücke der paläozoischen Kordillere heute wieder zutage.

1) Crarkit, J. M. Fosseis Devonianos do Parana. Servic. geolog. é mineralog. do Brasil. 1913. 
KeIDec hat neuerdings die älteren Beobachtungen ergänzt und umgedeutet (Memor. 1916, S. 75). Daraus erhellt, daß Vorkordillere und Sierra de la Ventana diesem einheitlichen paläozoischen Gebirgsssystem angehören, das möglicherweise seine Fortsetzung im Kapgebirge Südafrikas findet. Im einzelnen bleibt aber im Bau dieser alten Gebirge noch vieles aufzuklären. In der Sierra de la Ventana sind die glazialen Bildungen des Perm überschoben von intensiv gefalteten älteren paläozoischen Schichten, und auch in der Vorkordillere wiederholen sich die glazialen Ablagerungen in Schuppen mit den älteren Sedimenten. An einer anderen Stelle liegt dagegen nach $K_{\text {EIDEL }}$ die permische Grundmoräne auf einer geschliffenen und gekritzten Oberfläche über aufgerichteten Devonschichten.

Es ist KaIDELs großes Verdienst, nicht nur zuerst die permische Vereisung einwandfrei in Südamerika nachgewiesen, sondern auch die überaus große Übereinstimmung in der Gliederung der permischen Ablagerungen mit denen Indiens und Südafrikas gezeigt zu haben. Er beobachtete in der Vorkordillere zwei glaziale bzw. fluvioglaziale Horizonte, die durch kohlige Schiefer und Sandsteine getrennt sind und über denen Sandsteine und kohlige Schiefer mit Gangamopteris und Glossopteris folgen. Die Schiefer und Sandsteine parallelisiert KEIDEL mit den Talchirschichten und dem unteren Teil der Eceaserie, während die pflanzenführenden Schichten der Karharbaristufe in der Gondwanaserie entsprechen dürften. Auch die von anderen Punkten der Vorkordillere und aus den pampinen Sierren bekannt gewordene Gondwanaflora spricht für eine ähnliche Gliederung dieser Bildungen wie in Indien und Südafrika. Fine so weitgehende Ubereinstimmung im Habitus, in Gliederung und. Flora dieser Bildungen ist aber nur erklärlich, wenn wir annehmen, daß sie auf ein und derselben einheitlichen Kontinentalmasse gebildet wurden, die zu dieser Zeit große Teile von Südamerika und Afrika miteinander verband ${ }^{1}$ ). An anderer Stelle entdeckte KEIDEL zwei marine Einschaltungen in der glazialen Serie, die ältere mit Euomphalus und Pleurotomaria, die jüngere mit $S_{p}$ iriferen, die er mit den marinen Einschaltungen in den glazialen Bildungen Australiens und Tasmaniens vergleicht.

Auch die späteren permotriadischen terrestrischen Ablagerungen zeigen noch große Ähnlichkeit mit denen Südafrikas. Sie sind in Südbrasilien und Uruguay besonders mächtig entwickelt und früher allgemein unter dem Namen Santa Catharina Formation zusammengefabt worden. Ihre Gliederung hat in den letzten Jahren erhebliche Fortschritte ge-

1) Wenn neuerdings Sokraks (Das Problem der Ozeane und Kontinente Stuttgart, 1917) wieder versucht, auch für den Atlantischen Ozean die Persistenz zu beweisen und dabei glaubt, den Gondwanakontinent mit wenigen Worten abtun zu können, so scheint mir ein solches Verfahren wenig glücklich. Ein so schwieriges allgemeines Problem, wie die Frage nach der Persistenz der Ozeane läßt sich nur unter Würdigung aller Daten behandeln, nicht aber unter Herausgreifen einiger weniger Punkte. 
zeitigt. In Uruguay hat sich namentlich WALTHER (31) um sie verdient gemacht. Er hat seine Ergebnisse kürzlich zusammengefaßt und es läßt sich von der Gliederung und Parallelisierung mit den entsprechenden Ablagerungen Brasiliens und Südafrikas folgendes Bild gewinnen:

Sudafrika

Trias $\left\{\begin{array}{l}\text { Volcanic Beds } \\ \text { Stormberg } \mathrm{S} .\end{array}\right.$

Perm $\left\{\begin{array}{l}\text { Beaufort S. } \\ \text { Ecca S. }\end{array}\right.$
Uruguay u. S. Brasilien

Nelaphyrdecken im Territ. Misiones Sao Bentosandstein

Estrada Nova Schicht: Sandst., Schiefertone m. Kalk- u. Dolomiteinlagerungen, Kieselkonkretionen.

Bituminöse Iraty-Schiefer m. Mesosaurus u. Stereosternum (Sandst., Ton u. Quarzitschiefer m. Tubarao Gerölleinlagerungen, Orleanskonglomerat in S.-Brasilien.

Auch im argentinischen Territorium Misiones, das zwischen den Flüssen Paraná und Uruguay weit nach Norden vorspringt, sind die höheren Schichten der permotriadischen Serie entwickelt. Hier sind es namentlich die den Sao Bentosandstein überlagernden, aber gleichzeitig damit entstandenen Melaphyrdecken, die weithin das Landschaftsbild bedingen und die gewaltigen Wasserfälle der Nebenflüsse des Paraná, des Rio Iguazú und Nacunday verursachen. Die Melaphyrdecken sind von HANSEN (21) eingehend petrographisch untersucht worden. Dichter Melaphyr wechselt mit Mandelsteinen, die den alten Oberflächen der übereinander geflossenen Decken entsprechen.

Die Geschichte der brasilianischen Masse während des mittleren und jüngeren Mesozoikums bedarf noch in vielen Punkten der Aufhellung. Die Sedimentation scheint längere Zeit unterbrochen gewesen zu sein, und setzt erst in der Kreide wieder ein, vielleicht schon im Anschluß an die ersten orogenetischen Bewegungen, die zur Entstehung der heutigen Kordillere führten.' 'Diese Bewegungen griffen, wie wir eingangs gesehen haben, schlieblich auch auf die brasilianische Masse über und hoben in den pampinen Sierren Teile des alten Untergrundes wieder heraus. Auch die Erforschung dieser Gebirgszüge hat wesentliche Fortschritte gemacht. Rasmos (13) hat die Ergebnisse für die nördlicheren Gebirge neu zusammengefaßt; diese decken sich vollkommen mit meinen früheren Darlegungen über die südlicheren pampinen Gebirge (vgl. Geol. Rundsch. 1912). RAsmus' eigentliches Untersuchungsgebiet war die Sierra del Aconquija in der Provinz Tucuman. Sie gehört in geologischer Beziehung bereits zu den pampinen Sierren, obwohl sie nach Norden ihre unmittelbare Fortsetzung in den Vorkordilleren der Provinzen Salta und Jujuy findet. Fossilführendes Paläozoikum fehlt in 
ihr wie in allen Gebirgen der Pampa, und die tertiären Konglomerate der Kalchaquischichten sind nicht mit eingefaltet, sondern legen sich auf die gehobenen oder nur schwach verbogenen Rumpfflächen über den aufgerichteten alten Gesteinen auf. Dieses alte Gebirge zeigt sowohl in Beschaffenheit und Gliederung seiner stark veränderten Schichten als auch durch die gewaltigen Granitintrusionen, von denen das Massiv des Aconquija gebildet wird, große Ubereinstimmung mit den südlicheren Sierren. Wenn aber Rasmus seiner Auffaltung ein kaledonisches Alter zuschreiben will, so stimmt das wenig überein mit KeIDELS Beobachtungen, nach denen in den nördlicheren Vorkordilleren oberes Kambrium diskordant über den alten Gesteinen folgt. Überhaupt scheint mir die Übertragung der auf der nördlichen Halbkugel genau zeitlich und örtlich festgelegten Bezeichnungen kaledonisch und variszisch auf die Südhalbkugel wenig angebracht. Permotriadische Sedimente fehlen in der Sierra del Aconquija ebenso wie paläozoische. Unter den jungen Bewegungen, die das alte Gebirge stark herausgehoben und. dem andinen System angegliedert, unterscheidet RAsmus auf Grund morphologischer Befunde zwei Phasen.

Die südwestlich von dem eben besprochenen Gebirge in der Provinz Rioja gelegene Sierra de la Famatina (23) hat BoDENBENDER eingehend untersucht. Sie gehört geologisch zu den Vorkordilleren. In ihr ist fossilführendes Paläozoikum entwickelt, und auch die jungen tertiären Bildungen sind von der Andenfaltung ergriffen und mit den alten Gesteinen eingefaltet. Auch die permotriadische Schichtserie ist in der Sierra de la Famatina vorhanden. Der Kreide-Tertiärgrenze gehören mergelige Ablagerungen mit einer brackischen Fauna an, die auch weiter im Norden in Catamarca auftretenden Sta. Maria Schichten. Sie zeugen, wie ähnliche Bildungen weiter im Süden entlang dem Ostrand der Kordillere, davon, daß zu Beginn der Auffaltung dieses Gebirges entlang seinem Ostrand eine Senkung stattfand, sich eine Vortiefe herausbildete, gegen die hin die Faltung erfolgte, die aber schließlich von ihr auch ergriffen und dem Gebirge in Gestalt der Vorkordilleren angegliedert wurde. Wie in der Sierra del Aconquija und vereinzelt anch in den südlicheren pampinen Sierren, so setzt auch in der Famatina im Anschluß. an die Gebirgsbildung effusive vulkanische Tätigkeit ein. Eng verbunden mit den Daziten, Andesiten oder trachytischen Gesteinen ist die reiche Gold- und Kupfererzführung dieses Gebirges.

STAPPENBECK (3) verdanken wir eine geologische Übersichtskarte der Cordillera del Plata, des südlich des Rio Mendoza gelegenen Endes des östlichen Teils der Vorkordillere von Mendoza. Er verwertet hierbei zum erstenmal die Ergebnisse der mikroskopischen Untersuchung der von ihm früher in den Vorkordilleren von Mendoza und San Juan, sowie dem ihr im Osten vorgelagerten, aber bereits zu den pampinen Sierren gehörigen Pié de Palo gesammelten Gesteinen (10). Durch Granitintrusionen hochgradig veränderte, alte Schiefer und Grauwacken, die 
kontinentale Serie mit mächtigen Porphyrit- und Quarzporphyrbildungen in der Permo-Trias, pflanzenführende, bituminöse Rhätschiefer und ziegelrote Sandsteine und Konglomerate der" Kreide, ja selbst noch die Konglomerate, Sandsteine und Tuffe des Tertiärs, sind von der nach Osten übergreifenden Andenfaltung ergriffen und in weite Sättel und Mulden gelegt, die aber vielfach wieder durch Verwerfungen zerstückelt sind. Ja selbst die diluvialen Aufschüttungen am Rande des Gebirges sind stellenweise noch von den gebirgsbildenden Vorgängen in Mitleidenschaft gezogen. Südlich des Rio Mendoza taucht die östliche Antiklinale der Vorkordillere unter die sich mantelförmig auf sie auflegenden Aufschüttungen der Pampa. Der Co. Cacheuta wird von einem jungen, im Kern. dieser Antiklinale aufgedrungenen Granitstock gebildet.

Ehe wir uns nun den eigentlichen Kordilleren zuwenden, muß ich noch kurz auf die jüngsten kontinentalen Sedimente im Bereiche des alten Kontinents, die Bildungen der argentinischen Pampas zu sprechen kommen. KeIDex (2) hat sich in seiner Arbeit über die Sierra de la Ventana eingehend mit der Frage des argentinischen Löß beschäftigt und kommt dabei zu dem Ergebnis, daß dieser sowohl nach seinem Ursprung als auch nach seiner Entstehung recht verschieden von den europäischen Lößbildungen ist. Hierbei faßt KEIDEL den Begriff Löß sehr weit, er versteht darunter alle feinkörnigen mergelig-lehmigen Bildungen der Pampa. Er kommt daher auch zu dem Ergebnis, daß die Lößbildung in Argentinien bis ins Tertiär zurückreiche, während wir in Europa den Begriff Löß auf ganz bestimmte Sedimente ursprünglich äolischer Entstehung von diluvialem Alter beschränken. 、Es zeigt sich aber auch in Argentinen immer deutlicher, daß sich in der Pampasformation ältere lehmige. Ablagerungen von vorwiegend roter Farbe scharf trennen lassen von den jüngeren Bildungen von brauner oder gelber Farbe. Daß aber die älteren Pampaslehme in der Tat dem Tertiär angehören, darüber kann kein Zweifel mehr bestehen. Das beweisen nicht nur KeIDELs morphologische Beobachtungen in der sierra de la Ventana, sondern auch meine Befunde am Rande der Sierra de San Luis $^{1}$ ), wo die diluvialen Terrassen in die älteren roten Bildungen eingeschnitten sind. Am deutlichsten erhellt aber das tertiäre Alter dieser Bildungen aus den. Untersuchungen WALTHERS am Uruguayufer des La Plata. Er konnte das hier schon von Darwix beobachtete Profil vollauf bestätigen, in dem marine Schichten vom Alter der pliozänen Paranastufe den roten tonigen Sanden der Pampasformation eingelagert sind. In der Tat scheint die Farbe ein vielfach brauchbares Kriterium für das Alter der Pampasschichten. Dozning (Revista Mus. d. La Plata, T. XIV, p. 181) wies zuerst darauf hin, daß die roten tonigen Sedimente

1) GertH, Enr. Constitución geologica, hidrogeologica y minerales de aplicación de la provincia de San Luis. Annal. Minist. Agricult. T. X.

Geologische Rundschau, XII. 
Verwitterungsprodukte aus Zeiten darstellen mïssen, in denen noch ein warmes, tropisches Klima herrschte, während die Farbe und chemische Zusammensetzung der braunen und gelben schließen läßt, daß sie unter kälterem Klima gebildet wurden. Auch die Gliederung der Pampasbildungen im einzelnen ist weiter gefördert worden. Am Rande der Sierra de.Córdoba haben Frengueldi (26) und Casteldano (33) teils Bohrungen, teils die natürlichen Aufschlüsse an den Flußufern untersucht und sind im wesentlichen zu übereinstimmenden Resultaten gekommen. Über den roten Ablagerungen des Pampeano inferior folgen hier am Gebirgsrande vier fluviatile Einschaltungen, die durch verschieden mächtige Lößbildungen voneinander getrennt werden. Die Parallelisierung dieser Schichten mit den Stufen Ameghinos, die auf den verschiedenaltrigen marinen Transgressionen an der Küste basieren, scheint mir dagegen noch etwas unsicher. CAstellano stützt sich dabei neben den infolge der Spärlichkeit der Überlieferung immer leicht irreführenden paläontologischen Befunden, besonders auf die in den diluvialen Bildungen auftretenden Lagen von verwitterter vulkanischer Asche. Namentlich eine solche Schicht von grünlicher Farbe, von einer basischen Asche herrührend, die in den Lößbildungen zwischen der dritten und vierten fluviatilen Einschaltung von unten auftritt; scheint im Osten des Landes eine ziemlich allgemeine Verbreitung zu besitzen (Lucanense Ameghinos). Ob aber diese Aschenbänder sich nach Westen bis an den Kordillerenrand werden verfolgen lassen, wie Domring vermutet, und eine Parallelisierung mit den glazialen Bildungen ermöglichen werden, scheint mir fraglich. In den Profilen der bis über $1000 \mathrm{~m}$ tiefen Bohrungen in den Senken zwischen den pampinen Sierren der Provinz San Luis haben sich weder Aschenbänder noch fluviatile Einschaltungen gefunden, die mit denen der Provinz Córdoba verglichen werden könnten. Letztere sind vermutlich nur am Rande des Gebirges entwickelt, während in größerer Entfernung von diesem auch in den Pluvialzeiten die Sedimentation ursprünglich eine vorwiegend äolische war; dann allerdings vielfach eine Umlagerung durch fließendes Wasser erfolgte. Daß die Löße der jüngeren Pampasbildungen in Argentinien gerade wie auf der Nordhalbkugel ursprünglich äolischer Entstehung waren, beweist unzweideutig ihr Vorkommen bis auf die Höhen der pampinen Sierren. Aber mit KeIDEL müssen wir annehmen; daß die Lößmassen weniger aus eigentlichen Moränen als aus den gewaltigen fluvioglazialen Schotterablagerungen am Fuße der Kordillere ausgeblasen wurden. Hierdurch erklärt sich auch die häufig mehr flugsandähnliche Beschaffenheit des argentinischen Löß. Ebenso hat die abweichende chemische Beschaffenheit, wie die durch MrIGEN ${ }^{1}$ ) ausgeführten Analysen gezeigt haben, ibre Ursache in der Verschieden-

1) Mergen, W. und Werling, P. Über den Löß der Pampaformation Argentiniens. Ber. naturf. Gesellsch. Freiburg i. B, 1916. 
heit der Ursprungsgesteine. Das Überwiegen jungvulkanischer Bildungen in den Kordilleren und die verhältnismäßig geringe Verbreitung von Kalkgesteinen bedingt den geringen Gehalt an Karbonaten und den verhältnismäßig hohen Tonerdegehalt des argentinischen Löß.

Wenden wir uns nun der eigentlichen Kordillere zu. Auch hier hat die Durchforschung gewaltige Fortschritte gemacht, namentlich in Neuquen und im Süden der Provinz Mendoza. Doch liegen bis jetzt nur Teilresultate vor. Gromber (18) hat seine Ergebnisse über die Stratigraphie des Doggers zusammengefaßt. Daraus erhellt, daß sich der Dogger in der andinen Geosynklinale mit ähnlicher Gliederung und Fauna seiner Ablagerungen, wie sie schon lange vom Espinazitopa 8 in der Provinz Mendoza bekannt waren, bis nach Neuquen nach Süden erstreckt.

Das Problem der St. Jorgestufe (Rocaschichten), die den Schlüssel bilden für die jüngere Geschichte Patagoniens und der argentinischen Kordillere, ist von WINDHAUSEN erneut auseinandergesetzt worden, aber eine endgültige Klarheit in dieser so wichtigen Frage ist immer noch nicht erreicht. Hier muß erst durch eine Revision der aus diesen Schichten beschriebenen Fossilien und eine gründliche Bearbeitung des zahlreichen neueren Materials eine sichere Basis geschaffen werden, denn wohl nirgends in der paläontologischen Literatur herrscht eine so heillose Verwirrung, wie bei den Beschreibungen der Fossilien von Roca. In seiner klaren Zusammenfassung unserer Kenntnisse über die jüngeren Sedimente Patagoniens wies Wilckens ${ }^{1}$ ) auf die Beziehungen der Fauna der St. Jorgestufe mit den. Schichten mit Lahillia Luisa hin, die in Südpatagonien bei Ultima Esperanza unmittelbar über senonen Ablagerungen folgen und sich von diesen nur durch das Fehlen der Cephalopoden unterscheiden. WINDHAUSEN glaubte demgegenüber den rein tertiären Charakter. der St. Jorgestufe betonen zu müssen. Er trennt sie scharf ab von den Schichten mit Lahillia Luisa und sieht in ihrer Fauna den Ausgangspunkt für die Faunen der späteren marinen Transgressionen in Patagonien. Diese Auffassung läßt sich schlecht in Einklang bringen mit dem Fund typischer Kreidetrigonien an den nördlichsten bis jetzt bekannten Vorkommen der St. Jorgestufe in der Provinz Mendoza durch den Verfasser dieser Zeilen ${ }^{2}$ ). Es scheint sich vielmehr bei der St. Jorgestufe um eine letzte Transgression des antarktischen Kreidemeeres zu handeln, die von Süden nach Norden erfolgte und zeitlich etwa der dänischen Stufe entsprechen dürfte ${ }^{3}$ ). Sie ergoß sich in eine Vortiefe,

1) WiLckens, O. Die Meeresablagerungen der Kreide- und Tertiärformation in Patagonien. N. Jahrb. f. Mineralogie usw. Beil.-Bd. XXI. 1905.

2) Fritzsche, H. Eine Fauna aus Schichten der Kreide-Tertiärgrenze in der argentinischen Kordillere des südlichen Mendoza. Centralbl. f. Mineral. usw. 1919.

3) S. a. WrLokens, O. Beiträge zur Pala eontologie von Patagonien. N. Jahrb. f. Min. usw. 1921, Bd. I. 
die sich zu Beginn der Andenfaltung entlang dem Ostrande dieses Gebirges herausbildete und gegen die hin dann die Faltung erfolgte. Auch mit der Rekonstruktion der Ausdehnung dieser Transgression an der Kreide-Tertiärgrenze, wie sie WINDHausen gibt, können wir uns nicht in allen Punkten einverstanden erklären. Aus der Tatsache, daß wir aus dem Innern Mittelpatagoniens die St. Jorgestufe noch nicht kennen, ist nicht ohne weiteres zu schließen, daß sie dort nicht zur Ablagerung kam; sie kann vielmehr teils wieder der Abtragung anheimgefallen, teils aber auch bei der noch so unvollkommenen Durchforschung dieser Gebiete unserer Kenntnis entgangen sein. Die Zerstückelung der patagonischen Masse durch Verwerfungen, denen WINDHAdSEN eine so große Rolle für die Begrenzung der marinen Transgressionen zuschreibt, dürfte diese älteste, im Beginn der Andenfaltung erfolgte, noch wenig beeinflußt, sondern erst später eingesetzt haben. So wird auch die weitere Ausdehnung einer westnordweststreichenden Verwerfung, die den Golf der Rocaschichten im Rio Negrogebiet gegen Süden begrenzen soll, durch die Untersuchungen Wrohmanns (8) auf dem Südufer des Flusses nicht bestätigt. Die Hauptölgebiete Argentiniens, die die alte brasilianische Kontinentalmasse randständig umziehen, stehen ebenfalls mit den besprochenen Schichten in engster Beziehung, denn in den Olhorizonten in Comodore Rivadavia haben sich Versteinerungen der Schichten mit Lahillia Luisa gefunden.

Im einzelnen ist unsere Kenntnis von der Gliederung und Verbreitung der Ablagerungen in Nordpatagonien besonders durch die Arbeiten WICHMANNs ( 7,8 u. 28 Zusammenfassung) gefördert worden. Aus ihnen erhellt, daß der alte Untergrund in Patagonien an vielen Stellen unter den jungen Bildungen zutage tritt. Er besteht wie die ältesten Bildungen der pampinen. Sierren aus stark umgewandelten. Schiefern und Granitintrusionen. Über seine Struktur läßt sich noch kein sichéres Bild gewinnen, da die Streichrichtung der Schichten häufig wechselt. Die Oberfläche dieser alten Bildungen zeigt erhebliche Höhendifferenzen, diese und spätere Dislokationen bringen es mit sich, daß wir die mesozoischen und tertiären Bildungen nirgends in vollständiger Serie antreffen. Die älteste von diesen ist die Quarzporphyr-Formation, die für den ganzen südlichen Teil Südamerikas charakteristisch ist. Sie läßt sich von den argentinischen Vorkordilleren längs des ganzen Ostrandes der Anden nach Süden bis in die äußerste Spitze des Feuerlandes verfolgen. Aus den Kordilleren, in denen sie die Basis des mesozoischen Sedimentationszyklus der andinen Geosynklinale bilden, reichen sie in Patagonien nach Osten bis an die atlantische Küste. Sie ist von triadischem Alter, denn am Kordillerenrande in der Provinz Mendoza sowohl wie an der atlantischen Küste im Territorium Chubut wird sie von rhätischen Schiefern überlagert. Über ihr klafft in Patagonien, ebenso wie im Norden im Bereich der brasilianischen Masse, über den 
permotriadischen Bildungen eine Lücke in der Sedimentation. Diese setzt erst mit der mittleren Kreide wieder ein, und nun kommt die mächtige Sandsteinformation zur Ablagerung, die in Patagonien gewöhnlich als »Areniscas abigarradas « bezeichnet wird. Ihr Verbreitungsgebiet fällt ungefähr mit dem der Quarzporphyre zusammen. Thr oberer Teil wird vielfach auch Dinosauriersandstein genannt, er ist stellenweise mergelig und hat zahlreiche Reste von Tianosaurus und Dinosaurus geliefert (Pehuenche Amegrinos). In den obersten Lagen dieser mergeligen Schichten stellen sich dann zuweilen lakustre und schließlich brackische Bildungen ein, die ersten Vorboten der marinen Transgression der St. Jorgestufe, deren Ausdehnung und Bedeutung wir oben besprochen haben. Über den Rocaschichten der St. Jorgestufe folgen im Innern des Rio Negro-Gebiets wieder terrestre Ablagerungen, bunte Mergel und Sandsteine. Sie enthalten die Fauna der Pyrotherium-NotostylopsStufe Rotнs. Thre petrographische Ähnlichkeit hat dort, wo die trennenden marinen Schichten der St. Jorgestufe fehlen, vielfach zu Verweehslungen mit den liegenden Dinosaurierschichten geführt. So erklärt sich wohl das wiederholt behauptete Zusammenvorkommen der Dinosaurier mit der alttertiären Säugerfauna in Südamerika. Die Pyrotheriumschichten werden im Rio Negro-Gebiet von tuffreichen Bildungen überlagert, die WICHMANN als Capas de Chinchinales zusammenfaßt. Sie enthalten die Fauna der Colpodonstufe Rotus und sind möglicherweise bereits ein Äquivalent der ältesten Schichten der patagonischen Molasse, jener ausgedehnten marinen Transgression zur Miozänzeit. Über ihr folgt im Rio Negro-Gebiet der sogenannte Rio Negrosandstein, ein mächtiger Komplex terrestrisch-fluviatiler Entstehung, wie die eingelagerten Schichten mit Unio zeigen. Im Küstengebiet finden sich marine Einschaltungen sowohl an seiner Basis als auch in den oberen Partien. Sie führen die Fauna der ParanáStufe, der pliozänen Transgression im Parana-Gebiete, die, wie wir oben gesehen haben, auch ein Äquivalent der ältesten roten Ablagerungen der Pampa ist, des Pampeano inferior. Der Rio NegroSandstein wird im Norden von den. diluvialen Aufschüttungen der Pampa, im Süden von der patagonischen Geröllformation gleichen Alters bedeckt.

Nach dem gegenwärtigen Stand der Forschung ergibt die Gliederung und Parallelisierung dex känozoischen Ablagerungen Nordpatagoniens demnach folgendes Bild:

Terrestrische Bildungen: Marine Transgressionen:

Quartär: Patagonische Geröllformation (Jüngere Pampasbildnug im N)

Pliozän: Rio Negro-Sandstein Paraná-Stufe (Ältere Pampasbildung im N) 
Terrestrisohe Bildungen:

Miozän:

Oligozän :

Capas de Chichinales

(Colpodon-Stufe RotHs)

Eozän: Pyrotherium-Notostylops -

Schichten

Kreide: Dinosaurier-Sandstein

St. Jorge-Stufe

(Roca-Schichten)

Marine Transgressionen:

Patagonische Molasse

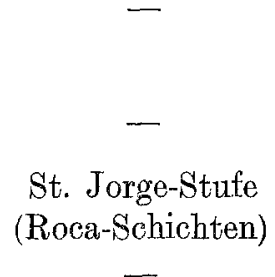

Zum Schlusse wollen wir uns noch dem äußersten Süden des Landes, dem Feuerlande zuwenden. Eine Untersuchung der ausgedehnten Torflager im Kordillerenvorlande veranlaßte BoNARELLI zu einer Zusammenstellung unserer Kenntnisse über den geologischen Bau des Feuerlandes, die von einer geologischen Utbersichtskarte begleitet ist. Er konnte sich hierbei besonders auf die grundlegenden Untersuchungen QueNsELs ${ }^{\mathbf{1}}$ ) über die patagonische Kordillere stützen. BonareuLI unterscheidet drei Einheiten in der Struktur des Feuerlandes, die vom pazifischen gegen den atlantischen Ozean hin aufeinander folgen und deren Alter in der gleichen Reihenfolge immer mehr abnimmt. Die Zone längs der pazifischen Küste wird durch den großen Andengranitbatholithen gebildet, der sich entlang der ganzen Westküste Patagoniens und des Feuerlandes hinzieht und in hochgradig veränderte Sedimentgesteine eingedrungen ist. Die metamorphen Schiefer treten sowohl im Westen im Dach des Batholithen als besonders an dessen Ostrand in einer kontinuierlichen Zone auf. Das Alter dieser Schieferformation ist immer noch nicht ganz festgelegt. Nur an der Tekenikabai wurde schon von DANA ein Belemnit (Helicerus fuegensis DANA) und dann neuerdings von Quensel Ammoriten gefunden, die oberjurassisches Alter besitzen. Die Hauptmasse der Phyllite, Grauwacken und Quarzite hält BoNaRelli dagegen für paläozoisch. Sie sollen von den mesozoischen Schichten der Tekenikabay durch feldspatführende Schiefer und sporadisch auftretende Mikrogranite getrennt werden. Die intensiv gefaltete Schieferzone wird gegen Osten begrenzt von einem Band von Quarzporphyren und Quarzporphyrtuffen, das sich ebenfalls von der Stateninsel bis nach Patagonien verfolgen läßt und hier mit den dort so verbreiteten Quarzporphyrmassen in Verbindung steht, denen, wie wir gesehen haben, ein frühmesozoisches Alter zukommt. Auf den Quarzporphyr folgt dann die mesozoische Serie der Kordillere. Auch diese Schichten sind stark gefaltet, und auch in ihnen treten kleinere Granitintrusionen auf. Fossilien haben sich bis jetzt nur am Mt. Tarn gefunden. Der chile-

1) Quenser, P. Geolog. petrogr. Stud. in d. patagon. Kordillere. Bull. geol. Institut of Upasal. Bd. XI. 1912. 
nische Geologe Fetsch ${ }^{1}$ ) hat dort neue Beobachtungen und Aufsammlungen gemacht und unterscheidet mehrere verschiedene Horizonte der oberen Kreide. Auf die mesozoische Zone folgen dann bis zur atlantischen Küste tertiäre Ablagerungen und diluviale Bildungen, die sich in ibrer Beschaffenheit und Gliederung eng an die Patagoniens anschließen. Borareli. können wir in ihrer Altersbestimmung, bei der er sich ganz an Ameghivo anschließt, nicht folgen.

1) Fetsch, J. Reconocim. geol. de los alrededores de Punta Arenas y de la parte N. O. de la Tierra del Fuego. Bolet. Inspee. geogr. y Minas. Bd. IV. Santiago di Chile. 1912. 